\section{SOI: $1.1 /$ TAS DOI: $10.15863 / \mathrm{TAS}$ International Scientific Journal Theoretical \& Applied Science}

Victor Aleksandrovich Melent'ev Philosophy Doctor, senior research associate Rzhanov Institute of Semiconductor Physics Siberian Branch of Russian Academy of Sciences (ISP SB RAS) melva@isp.nsc.ru

Valery Ivanovitsh Shubin Head of Department of Computer Networks ISP SB RAS woland@isp.nsc.ru

SECTION 4. Computer science, computer engineering and automation.

\title{
ON SCALABILITY OF COMPUTING SYSTEMS WITH COMPACT TOPOLOGY
}

Abstract: We investigate the potential parallelism of a computer system with the graph, which has the smallest diameter at the given order and degree. Formalized function of upper limitation on parallelism and topological scalability of the system in case of solving tasks with the complete graph of data. The analysis of these functions is given. Ways to increase the potential parallelism of compact computing system are considered.

Key words: parallel systems, scalability, compact topology.

Language: Russian

Citation: Melent'ev VA, Shubin VI (2016) ON SCALABILITY OF COMPUTING SYSTEMS WITH COMPACT TOPOLOGY. ISJ Theoretical \& Applied Science, 11 (43): 164-169.

Soi: http://s-o-i.org/1.1/TAS-11-43-30 Doi: crossef http://dx.doi.org/10.15863/TAS.2016.11.43.30

\section{О МАСШТАБИРУЕМОСТИ ВЫЧИСЛИТЕЛЬНЫХ СИСТЕМ С КОМПАКТНОЙ ТОПОЛОГИЕЙ}

Аннотация: Исследуется потенцииальный параллелизм вычислительной системы, граф которой при заданных порядке и степени имеет наименьший диаметр. Формализовань функции предельной распараллеливаемости и топологической масштабируемости таких систем при решении в них информационно полносвязных задач, дан анализ этих функций, рассмотрены пути повышения потенциального параллелизма компактных ВС.

Ключевые слова: параллельные системы, масштабируемость, компактная топология.

\section{Работа выполнена при поддержке Российского фонда фундаментальных исследований} (проект 14-07-00169a)

\section{Введение}

Глобализация информационных и вычислительных ресурсов требует организации эффективного управления соответствующими системами, как в аспекте их функциональной целостности, так и с позиций устойчивости функционирования. Устойчивость системы в процессе функционирования определяется адекватностью и своевременностью ее реакции на возникающие ситуации. Своевременность и рациональность управляющих воздействий во многом определяются сетью связи, являющейся, таким образом, неотъемлемой компонентой подсистем управления функционированием параллельных систем. В [1, с. 61] в рамках задачи генерации регулярных топологий $\mathrm{BC}$ по критерию задержек введено понятие компактного графа системы и определены условия компактности.

Как показано в [2, с. 120-124], определяющее значение в масштабировании параллельных систем и задач имеют используемая в ВС топология и реализованные в ней сетевые технологии, причем сетевая технология определяет пределы распараллеливания задач в предположении изоморфизма их информационных графов графу системы (как правило, для ограниченного круга задач в специализированных системах), или при полной связности графа ВС универсального назначения, что объективно реализовано быть не может из экономических соображений и из-за отсутствия технической возможности неограниченного наращивания числа портов в процессорах. Поэтому реальные пределы 
распараллеливания задач в системе составляют лишь некоторую, обеспечиваемую топологией системы, часть потенциала, который, в свою очередь, обеспечен используемой сетевой технологией.

Цель настоящей работы состоит в выявлении потенциала параллелизма и показателей масштабируемости вычислительных систем с компактными топологиями. В работе приведено описание модели параллельных вычислений, связывающей получение требуемых при реализации приложений ускорения и эффективности с предельным расстоянием между информационно смежными ветвями (достижимостью) и с их числом. Представлены способы описания лимитированных достижимостью и заданными значениями кратности отказов топологий ВС. Дано определение компактной топологии ВC, предложен формальный аппарат оценки ее потенциала параллелизма и масштабируемости таких систем.

\section{Основные положения}

Топологию вычислительной системы представим неориентированным графом $G(V, E)$ без кратных ребер и петель, где $V$ и $E$, соответственно, - множества вершин и ребер. Подграф $[a]_{i} \equiv G_{i}(u) \quad$ такого графа $G$, индуцированный множеством вершин, находящихся от вершины $a \in G$ на расстоянии ${ }^{1} i$, назовем $i$-окрестностью этой вершины. Множество вершин в $i$-окрестности $[a]_{i}$ будем называть $i$-окружением $\mathcal{N}_{i}(a), \quad$ число составляющих его вершин обозначим через $n_{i}(a)=\left|\mathcal{N}_{i}(a)\right|$. В дальнейшем при равенстве индекса $i$ единице 1 -окрестность $[a]_{1}$ будем называть просто окрестностью $[a] \equiv[a]_{1}, \quad$ а соответствующее такой окрестности 1-окружение — окружением $\mathcal{N}_{1}(a) \equiv \mathcal{N}(a)$ с числом вершин $n_{1}(a)=n(a)$.

Две вершины $u, v \in V$ графа $G(V, E)$ назовем эквивалентными $(u \sim v)$, если их окрестности совпадают: $u \sim v \Leftrightarrow[u]=[v]$. Понятно, что в этом случае окружения таких вершин также равны - $[u]=[v] \Leftrightarrow \mathcal{N}(u)=\mathcal{N}(v), \quad$ поэтому справедливо и $u \sim v \Leftrightarrow \mathcal{N}(u)=\mathcal{N}(v)$. Вершины $u$ и $v \quad$ графа $G \quad$ назовем дистанционно-эквивалентными $(u \simeq v)$, если все их $i$-окрестности вплоть до индекса $i$, равного диаметру $d(G)$ графа, попарно равномощны: $u \simeq v \Leftrightarrow n_{i}(u)=n_{i}(v), \quad i \leq d(G)$. Далее, если нет необходимости в сопоставлении разных графов, обозначение диаметра $d(G)$ графа сократим до $d$. Понятно, что $i$-окружения эквивалентных вершин

1 Расстояние $d(u, v)$ между вершинами $u$ и $v$ равно длине кратчайшего соединяющего их пути.

ISPC Education and Innovation, попарно равномощны - $u \sim v \Rightarrow n_{i}(u)=n_{i}(v)$, поэтому эквивалентные вершины дистанционно-эквивалентны ${ }^{2}-u \sim v \Rightarrow u \simeq v$. Если эксцентриситет ${ }^{3} \varepsilon(v)$ вершины $v$ меньше диаметра графа $d(G)$, то для всех $i$ таких, что $\varepsilon$ $(v)<i \leq d,-\mathcal{N}_{i}(v)=\varnothing$ и $n_{i}(v)=0$.

Объектом исследования в настоящей работе являются $\mathrm{BC}$ с компактной топологией, a предметом исследования - предельные возможности таких ВС в части организации в них подсистем с ограничениями сетевых задержек, т. е. предельные возможности компактных графов по наличию в них подграфов с лимитируемым значением достижимости. Методика проведенного исследования основана на формальном описании графа его проекциями. В связи с тем, что такая формализация графов является оригинальной и в научной литературе недостаточно широко представлена, в следующем разделе приведены краткие сведения о ней.

\section{Проективное описание графа}

Проекция $P_{i}(v)$ неориентированного простого графа $G(V, E)$ представляет собой $i$-уровневую конструкцию, на нулевом уровне которой расположена ракурсная (определяющая ракурс) вершина $v \in V$; порожденное ею на первом уровне окружение $\mathcal{N}(v)$, содержит $s(v)$ смежных $v$ вершин, принадлежащих окрестности $[v]$, а $i$-е уровни объединяют подмножества вершин, порожденные вершинами предшествующих уровней $[3$, с. 695; 4, с.170]. Отношение непосредственного предшествования/порождения вершин в проекции $P(v)$ есть отношение их смежности. Число порожденных на $i$-м уровне подмножеств соответствует числу $\left|V_{i-1}\right|$ вершин порождающего их $(i-1)$-го уровня. Упорядочив вершины по их непосредственному «предшествованию/порождению» от ракурсной $v$ до $j$-й вершины $i$-го уровня $v_{i j}$, получим маршрут $W\left(v-v_{i j}\right)=\left(v, v_{1 x}, \ldots, v_{i j}\right)$ из $v$ в $v_{i j}$, при этом в невзвешенном графе номер $i$ уровня равен длине пути из $v$ в любую вершину из подмножества $V_{i}$ находящихся на этом уровне вершин. Последовательность вершин, непосредственно предшествующих открывающимся скобкам от $v_{i j}$ до ракурсной вершины $v$, дает обратный маршрут $W\left(v_{i j}-v\right)=W^{-1}\left(v-v_{i j}\right)$.

В общем случае вершина $u \neq v$ в $i$-уровневой проекции $P_{i}(v)$ может повторяться $m$-кратно.

\footnotetext{
${ }^{2}$ Следует учитывать, что отношения эквивалентности могут связывать вершины лишь одного графа, тогда как отношения дистанционной эквивалентности могут быть отнесены к вершинам разных графов

3 Эксцентриситет $\triangle(v)$ вершины $v$ равен расстоянию от $v$ до наиболее удаленной вершины.
} 
Кратность $m(u)$ вершины $u$ в проекции и число описываемых ею простых цепей из ракурсной вершины $v$ в вершину $u$ равнозначны. Проиндексируем кратные вершины по порядку их размещения на уровнях «снизу-вверх» (по возрастанию номеров уровней) и «слева-направо» (для вершин одного уровня). Нулевое значение присвоим индексу экземпляра вершины, расположенного первым снизу и слева, назовем этот экземпляр оригинальным. Индексам остальных экземпляров $\quad u_{k} \in\left(u_{1}, \ldots, u_{m-1}\right)$ вершины $u$, присвоим соответствующие упорядочиванию значения из $1 \leq k \leq m(u)-1$. Эти экземпляры назовем реплицированными, или репликами. Уровни, на которых расположены экземпляры вершины $u$, также упорядочим по возрастанию: оригинальной вершине $u_{0}$ соответствует уровень $j_{0}=\min \left(j_{0}, j_{1}, \ldots, j_{m-1}\right)$, вершине $u_{m-1}-j_{m-1}$-й уровень. Числовые значения отдельных элементов множества $\left(j_{0}, j_{1}, \ldots, j_{m-1}\right)$ могут совпадать. Номер уровня $j_{0}$, на котором расположена оригинальная вершина $u_{0}$, имеет наименьшее значение, равное длине кратчайшего маршрута в множестве путей из $v$ в u. Таким образом, множество оригинальных вершин $i$-го уровня проекции $P(v)$ является $i$-окружением $\mathcal{N}_{i}(v)$ ракурсной вершины $v$ этой проекции.

\section{Понятие компактной вычислительной} системы

Одной из насущных проблем управления вычислительными системами (BC) является критичность ко времени реализации управляющих воздействий. Своевременность управляющих воздействий в процессе функционирования ВС во многом определяется компактностью ее топологии, обеспечивающей минимум транзитных узлов в межмодульных взаимодействиях. Компактные ВС определены в [1, с. 62] как системы, в основе интерконнекта которых лежит топология, заданная регулярным графом $G(V, E), \quad$ обладающим минимально возможным диаметром $d(G)$ при заданных значениях его порядка $n(G)=|V|$ и степени $s(G)$. Полученное в [1, с. 64] условие, связывающее порядок, степень и диаметр $n(s)$-компактного графа, имеет следующий вид:

$$
1+s \sum_{i=1}^{d-1}(s-1)^{i-1}<n(s) \leq 1+s \sum_{i=1}^{d}(s-1)^{i-1} .
$$

Для $s>2$ это условие представлено проще в [4, c. 96]:

$$
\frac{s(s-1)^{d-1}-2}{s-2}<n(s) \leq \frac{s(s-1)^{d}-2}{s-2} .
$$

Эти условия получены с использованием проективного описания топологий $\mathrm{BC}$, впервые предложенного в [3; 4]. Напомним в связи с этим, что проекция $P_{i}(v)$ неориентированного простого графа $G(V, E)$ представляет собой $i$-уровневую конструкцию, на нулевом уровне которой расположена ракурсная (определяющая ракурс) вершина $v \in V$; порожденное ею на первом уровне окружение $\mathcal{N}(v)$, содержит $s(v)$ смежных $v$ вершин, принадлежащих окрестности $[v]$, а $i$-е уровни объединяют подмножества вершин, порожденные вершинами предшествующих уровней. Отношение непосредственного предшествования/порождения вершин в проекции $P(v)$ есть отношение их смежности. Число порожденных на $i$-м уровне подмножеств соответствует числу $\left|V_{i-1}\right|$ вершин порождающего их $(i-1)$-го уровня. Упорядочив вершины по их непосредственному

«предшествованию/порождению» от ракурсной $v$ до $j$-й вершины $i$-го уровня $v_{i j}$, получим маршрут $W\left(v-v_{i j}\right)=\left(v, v_{1 x}, \ldots, v_{i j}\right)$ из $v$ в $v_{i j}$, при этом в невзвешенном графе номер $i$ уровня равен длине пути из $v$ в любую вершину из подмножества $V_{i}$ находящихся на этом уровне вершин. Последовательность вершин, непосредственно предшествующих открывающимся скобкам от $v_{i j}$ до ракурсной вершины $v$, дает обратный маршрут $W\left(v_{i j}-v\right)=W^{-1}\left(v-v_{i j}\right)[7$, c. 24].

В данной работе мы рассматриваем компактные графы предельного при заданных $d$ и $s$ порядка $N_{d}(s)$ (далее такие гипотетические ${ }^{4}$ графы мы называем предельно компактными и обозначаем далее $\left.G_{d}(s)\right)$ :

$$
N_{d}(s)=1+s \sum_{i=1}^{d}(s-1)^{i-1}=\frac{s(s-1)^{d}-2}{s-2} .
$$

Отметим основные свойства такого графа и его проекций:

1. Обхват предельно компактного графа $g\left(G_{d}(s)=2 d+1\right.$.

Случай с $n(s)=s(s-1)^{d} /(s-2)$ соответствует $n(s)$ компактному графу с максимально возможным обхватом $g(G)=2 d+1$ (если такой граф существует) и отсутствию в любой из его проекций вплоть до $d$-уровня кратных вершин ${ }^{5}$. Попытки синтезировать граф с меньшим, чем $2 d+1$, обхватом приводят к появлению кратных вершин на уровнях $i<d$. При этом $d$-уровневые проекции с кратными вершинами теряют вершинную полноту, что увеличивает эксцентриситеты этих ракурсных вершин, а следовательно, и диаметр графа, в результате чего граф потеряет свойство компактности. Таким образом, $n(s)$-компактных графов $c$ $n=s(s-1)^{d} /(s-2) \quad$ и обхватом $g(G)<2 d+1$ не существует [5, с. 97].

\footnotetext{
4 Предельно компактный граф не всегда может быть построен с точностью до порядка $N_{d}$ [1], поэтому мы говорим здесь о нем как о гипотетическом.

${ }^{5}$ Кратными в проекции $P_{d}(v)$ называют перечисленные в ней повторяющиеся вершины.
} 
2. Минимальное число уровней, необходимое для полноты ${ }^{6}$ описания предельно компактного графа $G_{d}(s)$ любой его проекцией, на единицу превышает диаметр этого графа.

3. В любой проекции предельно компактного графа вплоть до уровня с номером, равным его диаметру $d$, реплики ${ }^{7}$ вершин отсутствуют. Уровень $i=d+1$ проекции, превышающий диаметр $d$ на единицу, содержит только реплики вершин уровня $i=d$.

4. $d$-уровневая проекция предельно компактного графа $G_{d}(s)$ дает полное описание его остовного дерева $T\left(G_{d}(s)\right)$. Пары вершин смежных в проекции графа $G_{d}(s)$ уровней $d$ и $(d+1)$ определяют базис пространства его циклов $[6$, c. 183]. Все эти циклы имеют одинаковую длину, равную обхвату $g\left(G_{d}(s)\right)=2 d+1$, а мощность множества этих циклов (цикломатический ранг) - $\lambda\left(G_{d}(s)=\left|N_{d}(s) \cdot s / 2\right|\right.$ $N_{d}(s)+1=s(s-1)^{d} / 2$.

5. Все вершины предельно компактного графа $G_{d}(s)$ дистанционно эквивалентны. Напомним, что вершины $u$ и $v$ в графе $G$ дистанционно эквивалентны $(u \simeq v)$, если все их $i$-окружения вплоть до индекса $i$, равного диаметру $d(G)$ графа, попарно равномощны: $u \simeq v \Leftrightarrow n_{i}(u)=n_{i}(v), \quad i \leq d(G) ; \quad$ при $i$-окружение вершины $u$ - множество вершин, равноудаленных от $u$ на расстояние $i[7$, с. 25].

\section{Показатель топологической} масштабируемости вычислительных систем

Для оценки зависимости масштабируемости параллельных систем и решаемых на них задач от топологии используем модель параллельных вычислений, в которой параллельные приложения при условии бездефицитного быстродействия интерконнекта считаются неограниченно распараллеливаемыми. В реальности это условие выполнено быть не может, во-первых, из-за физической невозможности реализации полной связности большого числа объединяемых в системе процессоров и, во-вторых, из-за физических ограничений скорости распространения сигналов в сети связи. Поэтому предложенная в [2, с. 117] модель учитывает вносимые используемыми в системе топологией и сетевой технологией ограничения параллелизма. Формальная сущность этой модели заключается в коррелированности предельно допускаемого расстояния $\partial$ между информационно смежными в задаче процессорами измеряемой временем

\footnotetext{
6 Полная проекция несет в себе информацию обо всех вершинах и ребрах графа.

${ }^{7}$ Вершина $a_{i}$, где $i$ - наименьший в проекции номер уровня, на котором эта вершина появляется, носит название оригинальной, а все ее дубли на уровнях $j \geq i$ - реплики.
}

вычислительной сложностью $W$ этой задачи, измеряемым в информационных единицах объемом $Q$ подлежащих обмену данных и соответствующим требуемому ускорению минимальным числом задействованных в решении этой задачи процессоров:

$$
\partial_{S}(p)=\left\lfloor\frac{W}{s \cdot t_{N T}(Q / p)}\right\rfloor .
$$

Здесь $t_{N T}$ - известная для используемой в системе сетевой технологии (далее, $N T$ - Network Technology) зависимость задержки от объема передаваемой информации.

Из (1) ясно, что предельные значения достижимостей $\partial$ и числа процессоров $p$ для реализуемых в ВС с разными NT одних и тех же приложений различаются между собой тем значительнее, чем больше отличия в быстродействии используемых при этом NT. Таким образом, в зависимости от используемой сетевой технологии реализуемые в $\mathrm{BC}$ параллельные приложения можно классифицировать по значениям предельных для информационно смежных ветвей расстояний, допускающих потенциальную возможность достижения требуемого ускорения $S$, естественно - при топологической адекватности этих приложений системе.

Характеристику рассматриваемых здесь компактных ВC осуществляем показателем, введенным в [2, с. 138]:

$$
\mu_{\partial}(G)=\varphi_{\partial}(G) / n(G)
$$

Здесь $\mu_{\partial}(G) \quad-\quad$ топологическая масштабируемость ВС, заданной графом $G$, а $\varphi_{\partial}$ $(G)$ - плотность графа $\partial$-достижимости $G_{\partial}$

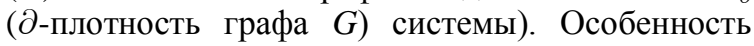
этого показателя состоит в предположении информационной полносвязности решаемых в системе задач и, таким образом, с его помощью оценивается нижний потенциал масштабирования.

Топологическая масштабируемость ВС с компактной топологией

Итак, в соответствии с упомянутой выше и используемой здесь моделью параллельных вычислений считаем, что реализованная в рассматриваемой системе NT позволяет классифицировать набор решаемых на ней задач по значениям допускаемой для этих задач достижимости $\partial$. Область изменения последней определим при этом интервалом $1<\partial \leq d$, так как для любого связного графа $G$ с обхватом $\lambda(G)>3$ значения плотности $\varphi_{\partial}(G)$ при $\partial=1$ и $\partial \geq d$ известны: $\varphi_{1}(G)=2$ и $\varphi_{\partial}(G)=n(G)$.

Пусть при этом топология ВС задана предельно компактным графом $G_{d}(s)$ степени $s$ и диаметра $d$, проекция этого графа $-P_{d+1}\left(x_{0}\right)$; где $x_{0} \in V$ - ракурсная вершина проекции, $V-$ множество вершин рассматриваемого графа, 
индекс $d+1$ при проекции $P_{d+1}\left(x_{0}\right)$ указывает на число уровней в этой проекции (заметим, что согласно сформулированному выше свойству 2, проекция $P_{d+1}\left(x_{0}\right)$ графа $G_{d}(s)$ является полной).

В [8, с. 78] показано, что расстояние между двумя вершинами графа не превышает суммы номеров уровней описывающей этот граф проекции. Расстояние $d\left(x_{i}, y_{j}\right)$ между вершинами $x_{i}, y_{j}$, расположенными на соответствующих индексам уровнях $i, j \leq d(G) \quad d$-уровневой проекции $P_{d}(v)$ принадлежит интервалу значений от разницы $|i-j|$ номеров их уровней (если одна из них принадлежит пути из $x_{0}$ в другую) до их суммы $i+j$ (если этот путь проходит через ракурсную вершину $\left.x_{0}\right): \quad|i-j| \leq d\left(x_{i}, y_{j}\right) \leq i+j$. Промежуточные в этом интервале длины путей из $x_{i}$, в $y_{j}$ дискретны с шагом 2 от $|i-j|+2$ до $i+j-2$ (в зависимости от того, в какой из вершин совпадают пути из $x_{0} \quad$ в $x_{i} \quad$ и $y_{j}$ ). Для определенности далее считаем $j \leq i$, тогда при равенстве $j=i$ расстояние между вершинами $x_{i}, y_{j}$ становится максимальным, соответственно,

$$
2 \leq d\left(x_{i}, y_{i}\right) \leq 2 i \text {. }
$$

Заметим, что справедливость (3) в предельно компактном графе сохраняется только при $i \leq\lfloor d / 2\rfloor$.

Для случая $i \geq\lceil d / 2\rceil$, следует учесть, что обхват рассматриваемого здесь предельно компактного графа $-g\left(G_{d}(s)\right)=2 d+1$ (свойство 1), все фундаментальные его циклы по длине равны обхвату (свойство 4), а все вершины этого графа дистанционно эквивалентны (свойство 5). Соответственно, расстояния $d\left(x_{i}, y_{i}\right)$ в таком случае не превысят значений, определяемых интервалом:

$$
2 \leq d\left(x_{i}, y_{i}\right) \leq 2 d+1-2 i .
$$

Сравнивая интервалы в (3) и (4), определим номер уровня, на котором верхнее граничное значение интервала (4) гарантированно уменьшится, как минимум, до значения из (3): $2 i \geq 2 d+1-2 i$. Естественно, что все значения $i$ и дробей с числителями $i, d$ и $\partial$ при этом целочисленны, поэтому ниже рассмотрим случаи четного и нечетного диаметра $d$ предельно компактного графа.

1. Учитывая, что при четном диаметре $\lfloor d / 2\rfloor=\lceil d / 2\rceil=d / 2, \quad 2\lfloor d / 2\rfloor=2\lceil d / 2\rceil=d$, получим:

если $i<d / 2$, то $2 i<2 d+1-2 i$, и

$d\left(x_{i}, y_{i}\right)=\min \{2 i, 2 d+1-2 i\}=2 i$

если $i=d / 2$, то $2 i<2 d+1-2 i(d<d+1)$,

$d\left(x_{i}, y_{i}\right)=\min \{d, d+1\}=d$, и $d\left(x_{i}, y_{i}\right)=2 i$;

если $i>d / 2$, то $2 i>2 d+1-2 i$,

$d\left(x_{i}, y_{i}\right)=\min \{2 i, 2 d+1-2 i\}=2 d+1-2 i$ и

$d\left(x_{i}, y_{i}\right)<2 i$.
2. При нечетном диаметре $\lfloor d / 2\rfloor=\lceil d / 2\rceil-1, \quad 2\lfloor d / 2\rfloor=d-1, \quad 2\lceil d / 2\rceil$

$=d+1$. Из этого следует:

если $i<\lfloor d / 2\rfloor$, то $2 i<2 d+1-2 i$, и

$d\left(x_{i}, y_{i}\right)=\min \{2 i, 2 d+1-2 i\}=2 i$;

если $\quad i=\lfloor d / 2\rfloor, \quad$ то $2 i<2 d+1-2 i \quad(d-$

$1<d+2)$, и $d\left(x_{i}, y_{i}\right)=\min \{d-1, d+2\}=d-1$;

если $i=\lceil d / 2\rceil$, то $2 i>2 d+1-2 i(d+1>d)$,

и $d\left(x_{i}, y_{i}\right)=\min \{d+1, d\}=d$.

Обобщив приведенные выше соотношения для случая, когда расстояние между одноуровневыми вершинами предельно компактного графа не превысит удвоенного номера этого уровня, получим, что $d\left(x_{i}, y_{i}\right) \leq 2 i$, только если $i \leq\lfloor d / 2\rfloor$. Из этого ясно, что если заданное значение достижимости $\partial<d$, то расстояния между вершинами, расположенными на уровнях $i \leq\lfloor d / 2\rfloor$ проекции $P_{d+1}\left(x_{0}\right)$, не превысят этого значения, то $\forall x_{0}, x_{i}, x_{j} \in V$, $j \leq i \quad 2 \leq d\left(x_{i}, x_{j}\right) \leq \partial$, здесь $V$ - множество вершин графа $G_{d}(s)$. Понятно, что число таких вершин, расстояния между любой парой которых не превысит четного значения заданной достижимости $\partial-\partial$-плотность графа $G_{d}(s)$, составит

$$
\varphi_{\partial}\left(G_{d}(s)\right)=1+s \sum_{i=1}^{\partial / 2}(s-1)^{i-1}=\frac{s(s-1)^{\partial / 2}-2}{s-2} .
$$

При нечетном значении $\partial<d-1-$

$$
\begin{gathered}
\varphi_{\partial}\left(G_{d}(s)\right)=1+s \sum_{i=1}^{\lfloor\partial / 2\rfloor}(s-1)^{i-1}+(s-1)^{\lfloor\partial / 2\rfloor}= \\
=\frac{2\left((s-1)^{\lceil\partial / 2\rceil}-1\right)}{s-2} .
\end{gathered}
$$

Из (2), (5), (6) получим показатели масштабируемости $\mu_{\partial}\left(G_{d}(s)\right) \quad$ предельно компактных ВС для четных -

$$
\mu_{\partial}\left(G_{d}(s)\right)=\frac{s(s-1)^{\partial / 2}-2}{s(s-1)^{d}-2}
$$

и для нечетных -

$$
\mu_{\partial}\left(G_{d}(s)\right)=\frac{2\left(s(s-1)^{\lceil\partial / 2\rceil}-2\right)}{s(s-1)^{d}-2}
$$

значений требуемой достижимости $\partial<d\left(G_{d}(s)\right)$. Понятно, что при $\partial \geq d\left(G_{d}(s)\right)$ и при нечетном $\partial \geq d-1-\varphi_{\partial}\left(G_{d}^{n}(s)\right)=n$ и $\mu_{\partial}\left(G_{d}(s)\right)=1$.

Можно было бы предположить, что для компактных графов $G_{d}^{n}(s)$ с заданными диаметром $d$ и степенью $s$ и с порядком $n$, меньшим порядка $N_{d}(s)$ предельно компактного графа $G_{d}(s) \quad\left(N_{d-1}(s)+1 \leq n \leq N_{d}(s)\right), \quad$ значения 
плотности $\varphi_{\partial}\left(G_{d}^{n}(s)\right) \quad$ и $\quad$ значения

масштабируемости

$$
\mu_{\partial}\left(G_{d}^{n}(s)\right) \text { при }
$$

соответствующих значениях достижимости $\partial$ будут находиться в соответствующих интервалах:

$$
\begin{aligned}
& \varphi_{\partial}\left(G_{d-1}(s)\right)<\varphi_{\partial}\left(G_{d}^{n}(s)\right) \leq \varphi_{\partial}\left(G_{d}(s)\right), \\
& \mu_{\partial}\left(G_{d}(s)\right) \leq \mu_{\partial}\left(G_{d}^{n}(s)\right)<\mu_{\partial}\left(G_{d-1}(s)\right) .
\end{aligned}
$$

Однако, как мы видим из (5), (6), Ә-плотности $\varphi\left(G_{d-1}(s)\right)$ и $\varphi\left(G_{d}(s)\right)$ не зависят от диаметра $d$, пока достижимость $\partial$ не сравняется с ним. Поэтому $\partial$-плотность компактного графа $G_{d}^{n}(s)$ не зависит от порядка $n$ этого графа. Понятно, что масштабируемость компактного графа меняется при этом обратно пропорционально порядку $n$.

Из вышесказанного следует, что горизонтальное масштабирование ВC с компактной топологией не приведет к увеличению потенциала ее параллелизма для информационно полносвязных задач, расстояния между ветвями которых в условиях используемой в системе NT лимитировано достижимостью, меньшей диаметра графа ВС. Увеличение потенциала параллелизма такой ВС может быть достигнуто «реберным» ее масштабированием (увеличением степени графа) таким образом, чтобы уменьшенный в результате этого диаметр графа ВС соответствовал предельным расстояниям между ветвями решаемых на ней параллельных задач - при этом потенциал параллелизма системы повысится до предельного значения. Другой путь к достижению предельного потенциала параллелизма параллельной системы - увеличение быстродействия используемой $N T$ таким образом, чтобы значения требуемой для задач достижимости превысили диаметр ее графа.

В принципе, оба из указанных здесь путей повышения потенциала параллелизма применимы для ВС с любой топологией, однако наиболее эффективны они именно в компактных ВС, графы которых по определению таковых обладают минимально возможными для заданных порядка и степени диаметрами.

\section{Заключение}

В продолжение работы по исследованию топологической масштабируемости гиперкубической ВС [10, с. 122-129] проведено исследование топологии, обеспечивающей минимальное расстояние между процессорами системы при заданных значениях степени и порядка ее графа. Введено понятие предельно компактного графа ВС и получены функции предельного параллелизма и топологической масштабируемости параллельной системы с заданными значениями порядка и степени компактного графа.

Результаты работы могут быть полезными в сопоставлении топологий параллельных систем в отношении масштабирования таких систем и обеспечиваемого при этом потенциального параллелизма решаемых на них задач

\section{References:}

1. Melent'ev VA (2014) About topological compactness of computing systems. ISJ Theoretical \& Applied Science 11 (19): 59-65.

2. Melent'ev VA (2015) On topological scalability of computing systems // Upravlenie bol'simi sistemami, 2015, Issue 58, p. 115-143.

3. Melent'ev VA (2004) The formal rudiments of a bracket patterns' in graph theory // Proceedings of the II International Conference "Parallel Computations and Control Problems" PACO '2004. Moscow, 4-6 October 2004, p. 694-706.

4. Melent'ev VA (2005) Formalnyj podhod k issledovaniyu struktur vychislitelnyh sistem // Vestnik Tomskogo Gosudarstvennogo Universiteta, - 2005, №14, p. 167-172.

5. Melent'ev VA (2011) Restrictions on girths in compact graphs // Prikl. Diskr. Mat., 2011, supplement № 4, 96-98.

6. Harary F (1973) Graph Theory. M.: Mir, 1973. p. 300 .
7. Melent'ev VA (2015) Limit configuring of subsystems in hypercubic computing systems // Informacionnye tekhnologii i vychislitelnye sistemy, 2015, No. 2, p. 20-30.

8. Melent'ev VA (2002) The bracket Pattern of a Graph // The 6th International Conference on Pattern Recognition and Image Analysis: New Information Technologies, PRIA-6-2002, October 21-26, 2002, Proceedings, Velikiy Novgorod, p. 57-61.

9. Melent'ev VA (2010) An analytical approach to the synthesis of regular graphs with preset values of the order, degree and girth // Prikladnaya discretnaya matamatika, 2010, №2(8), p. 74-86.

10. Melent'ev VA, Shubin VI, Zadorozhny AF (2015) Topological scalability of hypercubic parallel systems and tasks. ISJ Theoretical \& Applied Science 11 (31): 122-129. 